\title{
Halalopathy: Revival of Miraculous Cure and Creation of Favourable Circumstances for Cancer Therapy
}

\author{
Jawad Alzeer* \\ Halalopathy, Swiss Scientific Society for Developing Countries, Zurich, Switzerland \\ *Corresponding Author: Jawad Alzeer, Halalopathy, Swiss Scientific Society for Developing Countries, Zurich, Switzerland.
}

Received: February 08, 2022; Published: March 03, 2022

DOI: $10.55162 /$ MCMS.02.021

\begin{abstract}
Curing diseases and achieving complete recovery is challenging particularly with complex diseases such as cancer, where complete remission is most likely the preferred option that can be achieved. The hope for a complete recovery is inspired by cancer patients who have successfully beaten cancer and achieved a complete recovery. Despite physicians referring to these stories as "miraculous" recoveries, nevertheless, they reveal that victory over cancer can be achieved. According to Halalopathy, a key role in complete recovery is played by setting up the favourable circumstances for the cure, a process that can be achieved by involving the patient in the therapeutic process. Entropy and potential energy are inversely proportional, and both are key elements in the process of prevention and cure. The catabolic reaction is driven by entropy, while the anabolic reaction is driven by potential energy. The stimulation of potential energy and the reduction of entropy contribute greatly to the activation of the immune system and thereby create the necessary circumstances required for effective prevention and therapy.
\end{abstract}

Keywords: Cancer; miraculous cure; potential energy; entropy; immune system

\section{Introduction}

Cancer is a complex disease that affects almost every family, and treatment options are limited to chemotherapy, radiation, and surgery [1]. The concept of a complete recovery is not feasible for most patients undergoing cancer treatment, indeed with those options, it is difficult to completely remove cancer cells from the body in the time frame required for treatment. In theory, if one cancer cell remained in the body may have the potential to regrow and cause cancer to reproduced. In the meantime, there are successful stories of defeating cancer in almost every society, however, the point of view and feedback from physicians with regards to the success stories is varied: some physicians do not believe that these people had cancer and therefore assume that there was a mistake in the diagnosis, others believe that the patients had cancer and managed to recover completely, thinking that something unusual happened to their immune system that worked more effectively than usual, and therefore refer to this kind of recovery as a "miraculous" recovery [2]. Scientifically speaking, a miracle is an event that cannot be repeated or reproduced. However, if one understands scientifically what happened and under what circumstances a complete recovery occurred, one can process the outcome knowledge and use it to reproduce the circumstances that could lead to a complete "miraculous" recovery [3].

The market is nearly saturated with cancer drugs and any new drug that is developed provides limited added therapeutic value compared to existing drugs for the same type of cancer [4]. The development of the "magic bullet," i.e., a drug that hits its target and provides complete therapy for cancer patients without harming or killing the patient, is still considered a major challenge in the scientific community [5]. The main objective of the manuscript is to improve the effectiveness of existing medications and promote the healing process towards a complete recovery. To achieve this objective, the patient must play a role and get involved in the healing process. The patient's will to cure can be activated and the most favourable circumstances can be created to ensure a more effective treatment. 


\section{The path from halalopathy to cancer therapy}

To understand the concept of halalopathy and to illustrate the reason for identifying halalopathy as a new model for prevention and cure, we will outline the process of identifying the key elements, in particular potential energy and entropy, as well as the rationalisation of cancer from a halalopathic perspective and the potential implementation of halalopathic principles in cancer therapy.

\section{Anabolic and catabolic processes in terms of entropy and potential energy}

In order to properly understand the role of favourable circumstances and their connection to the immune system, it was necessary to focus on the main biological processes closely associated with the development and healing of diseases and to define the key factors that determine the course and direction of these processes. In halalopathy, catabolic and anabolic processes were emphasized as the processes of choice, and their pathways in our body were evaluated, focusing on the chemical pathways involved in the regulation and control of both processes [6]. It is obvious that the catabolic reaction occurs spontaneously and without the need for much energy. In fact, only a minimum amount of energy is required to start the reaction, but an enormous amount of energy in the form of ATP is released throughout the catabolic process. The driving force that causes the reaction to proceed spontaneously is the rise in entropy during the course of the reaction. Entropy increases as macromolecules, which have a high potential and rich information content, breakdown into micromolecules, which have a lower potential and higher entropy. In the anabolic reaction, the process proceeds in the opposite direction to entropy, whereby small molecules with low potential are collected and joined together to form a macromolecule that has a higher potential with more accurate information. Thus, the anabolic reaction is non-spontaneous and as a whole is driven by potential energy, while the catabolic reaction is driven by entropy. If insufficient energy is available, the anabolic process slows down and may not be able to continue the growth or synthesis of vital macromolecules that are important for maintaining information, regulation, and communication necessary for cell division and sustainability. The catabolic reaction proceeds regardless of the amount of potential energy available.

Potential energy represents the ability to do work and can be expressed in various forms. However, when it is dispersed or suppressed, it turns into entropy, which lacks the ability to do work, and when it accumulates in the body, it is harmful and can lead to major disorders and consequently the development of diseases. The enrichment and availability of potential energy play a role in promoting the anabolic process and activating the immune system, while the accumulation of entropy slows down the anabolic process and deactivates the immune system. Halalopathy as a new medical model is the first to address entropy as the main target for prevention and cure. Potential energy is inversely proportional to entropy, and therefore the main objective of Halalopathy is to rationalize the circumstances that can contribute to the accumulation of potential energy and reduction of entropy [7].

\section{Disease development and prevention from halalopathic perspective}

Numerous biological processes take place in the body around the clock, which are mainly biochemical reactions that take place in an appropriate balance to maintain the homeostasis of the body. For example, the body strives to maintain body temperature at $37^{\circ} \mathrm{C}$, and a drop or rise in body temperature is considered a defect, likewise, a drop or rise in blood pH, blood oxygen, or blood glucose levels, are defects that must be corrected immediately [8]. Normally, the immune system senses the defect and corrects it. However, if the defect is not fixed, it continues to accumulate in the body, increases disorder, and eventually leads to disease [9].

A defect is mainly caused by either a rise or drop in the rate of biochemical reaction, resulting in an up-or down-regulation of a biological process that is essential for maintaining the integrity of the body. If the defect cannot be repaired by the immune system, medical assistance becomes very necessary. Based on the diagnosis, the physician selects the proper medication that will either speedup or slowdown the process responsible for the defect. Generally, drugs do not trigger new processes in the body, they either speed-up a slow process or slowdown a speedy process without modifying the process as a whole [10]. 
In the body, it is important to know who primarily controls the rate of reaction and what circumstances can lead to a speed up or slowdown of the biological process. The brain plays a key role in controlling the body through the release of various neurotransmitters that either have an excitatory effect and speed-up the reaction process (e.g. dopamine) or an inhibitory effect and slowdown the reaction process (e.g. serotonin). The life span of these chemicals is less than 15 minutes, after which they are broken down into other metabolites [11]. If the body is under the influence of these chemicals for a longer period of time, diseases such as anxiety or depression may occur. The circumstances that cause anxiety are tension, worry, and fear, which are mainly situations relate to the future, while the circumstances that cause depression are grief, regret, heartbreak, pessimism, and despair, which are situations related to the past. In anxiety, the immune system operates in a disordered, uncontrolled mode, suggesting that potential energy is in a dispersed form, high entropy, and operating in a hunting mode in which the immune system is neither focused nor effectively productive, it is in a frightmode. Similarly, in depression, the potential energy is suppressed, high entropy, and the immune system is in flight mode. A healthy person maintains the immune system in the fight mode, which is the state in which the body is neither in fear nor in grief; it is the phase of high potential. Anxiety or depression is a long-term dispersion or suppression of the potential energy and should be considered very serious if not treated [12]. Therefore, people with such a condition need to see a physician who can help them regain control of the potential energy and keep the immune system in the fight mode. Otherwise, the immune system will ignore many defects that need to be repaired, and critical diseases such as cancer or other related diseases such as cardiovascular disease, diabetes, or Alzheimer's disease may develop if no action is taken [13-16]. Hence, if the physician is avoided, it is possible to regain the potential by avoiding/resisting the state of fear and/or grief and replacing it with the state of positivity, the state that is full of potential, optimism, joy, smiles, and hope; and if that still doesn't work, one can seek support, from friends who have a positive personality, who believe that with adversity comes lightness and relief. Meeting with a person who has a negative character is forbidden, as such a personality would complicate the situation (Figure 1) [17].

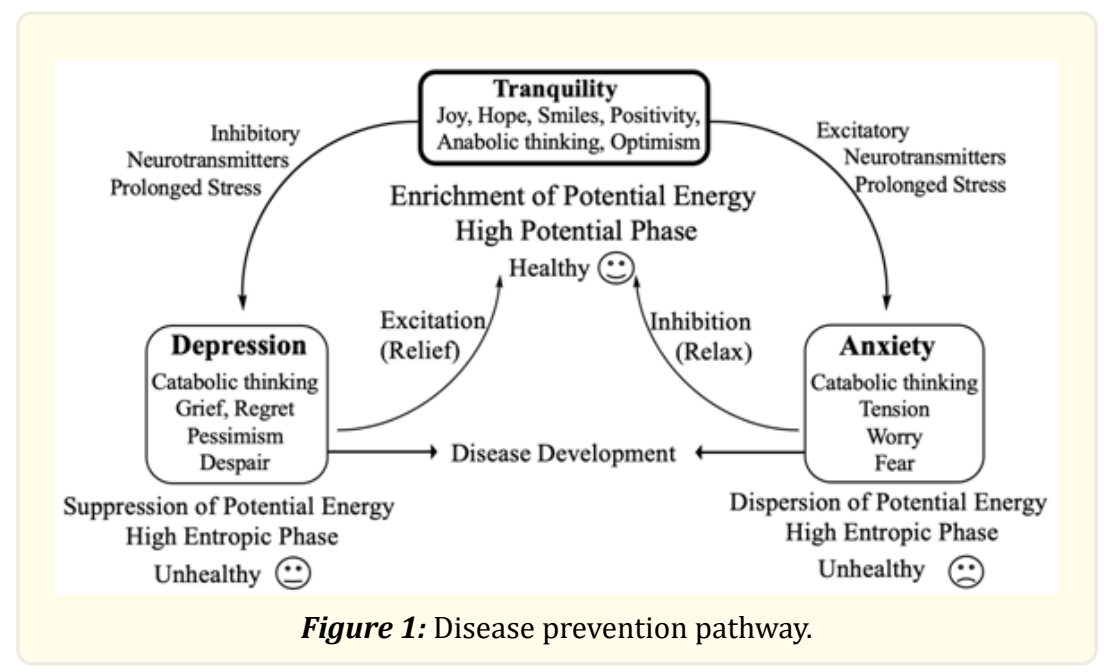

In fact, most diseases, including cancer, can be linked to anxiety or depression. Disease prevention can be enhanced by activating the immune system, which in turn can be achieved by maintaining the fight mode in an active state. Deactivation of the immune system is mediated by dispersion and/or suppression of potential energy, thereby reducing the potential for disease prevention and activating the flight/frightmode. The defect that occurs during flight/frightmode is not readily recognized by the immune system. If the defect is not corrected, it transforms the condition that was intended to be temporary into a permanent one that allows entropy to dominate, accumulate disorder in the body, and thus create favourable conditions for the development of diseases such as cancer. For this reason, anxiety and depression are key factors in the growth of cancer cells, and eliminating this condition will help to protect or prevent the progression of cancer. Consequently, there is a close relationship between mind, body, and disease, and creating favourable circumstances for the cure and activating the will to cure are essential for prevention and recovery. 


\section{Drug metabolism}

In cancer therapy, the pharmacokinetics of cancer drugs can shed light on why a complete recovery is not possible and why a complete remission is most likely the best option that can be achieved [18]. Pharmacokinetics is concerned with how drugs reach their site of action and are removed from the body. In fact, before a drug can reach its target cells and receptors to exert its effect, it must be effectively absorbed and then distributed to the desired site of action [19]. Metabolism and elimination of a drug may reduce either the amount or the duration of the effective action of the drug. Increasing the effective concentration of the drug or the duration of action may result in more effective treatment. However, since dose determines toxicity, it is important to carefully balance the therapeutic and toxic effects of the drug and reach a compromise between the two effects in order to achieve an acceptable recovery with the least toxic side effects [20]. Thus, the administered dose of the drug is not always sufficient to completely cure the patient, and increasing the dose leads to toxic effects, thereby making the balance between the therapeutic dose and the toxic effect of the drug very challenging. Generally, the body treats the drug as a foreign substance that is not compatible with the human body and must be removed quickly. Therefore, the administered drug is rapidly oxidized and/or conjugated with other molecules to make it more water-soluble and eliminated from the body, resulting in insufficient concentration and duration of action for most cancer therapies, thereby reducing its potential and ability to provide complete recovery.

\section{Miraculous recovery}

For the purpose of understanding how cancer survivors managed to achieve a "miraculous" recovery, many of these cases were studied, and as a result of the study, it was found that cancer survivors had not relied solely on the therapeutic effects of cancer drugs to achieve a complete recovery [21]. The most important aspect to consider was the incorporation of other values besides the therapeutic value, and in particular, they had replaced their old lifestyle with a new one, assuming that the old lifestyle was responsible for the development of cancer. By adapting and implementing the new lifestyle, they ensured that their behaviour and life performance were strictly compatible with the adapted lifestyle. Furthermore, these patients practiced sports such as yoga, meditation, mindfulness, and various spiritual practices. Clearly, from these successful stories, cancer therapy played a role, but the main role was played by the patient who managed to create the favourable circumstances required to achieve a complete recovery [22].

Halalopathy, a permissible medicine, was introduced to increase the effectiveness of existing drugs and promote the healing process towards complete recovery [23]. Halalopathy assumes that favourable circumstances play an essential role and are mainly related to the activation of the immune system [24]. Therefore, it was necessary to implement the key elements for the development of favourable conditions and to enrich the key factors needed for the activation of the immune system [25].

\section{Patient with cancer disease}

The moment the patient receives the news that cancer has been detected in the body, a state of anxiety and depression begins to prevail. As a result, the immune system is distracted and goes into fright/flight mode. If this mode is temporary, it is manageable, however, if it remains permanent, the patient who started with one disease, cancer, will have a total of three diseases upon hearing the news: anxiety, depression, and cancer [26-28]. When chemotherapy is administered while the immune system is in distraction mode, the therapeutic outcome is limited to the therapeutic effect of the chemotherapy or other therapeutic options, while other important effects related to potential energy sources are either dispersed or suppressed. These are absolutely not the appropriate and not favourable circumstances for healing, therefore it is important to activate the favourable circumstances necessary for healing before proceeding with treatment. Most cancer patients are in a highly entropic state [29], and therapeutic drugs are likely to be only partially effective in treating the disease; therefore, chemotherapy, radiation, and surgery can successfully remove most cancer cells. However, if one cancer cell remains in the body, it is very likely that cancer will grow again, thereby preventing a complete recovery. The key role in cancer treatment is primarily played by the immune system.

Communication between cells and with the brain is essential for keeping the body healthy. Cancer cells no longer respond to many 
of the signals that control cell growth and eventually escape programmed cell death, apoptosis, making them undetectable to the immune system. Immunotherapy is an innovative new approach to cancer treatment that uses the body's own defenses to fight tumours [30]. From a halalopathic perspective, it is important for more effective recovery to reduce entropy and enrich potential energy before and during treatment, which in turn can activate the immune system and trigger fight mode. One possible approach to activating the potential is to build a compatible system that allows the mind to be involved in the treatment process. A compatible system usually creates a highly ordered system with a high potential in which the communication between mind and body is intensified and enriched; moreover, since cancer cells are part of the body, the communication between mind and cancer cells could be reactivated. In this case, each organ of the body is treated as part of the whole, allowing the immune system to better recognize the cancer cells and subject them to either apoptosis or digestion by dendritic cells [31].

\section{Discussion}

The brain and its higher cognitive functions are central to health and therapy. The integration of mind, behaviour, and health is one of the greatest scientific challenges, yet effective integration would lead to a well-defined system. The mind can guide behaviour and health through well-organized, regulated, and connected systems. Cooperative interaction between the mind and the therapeutic drug through a well-defined and compatible system can create potential and deliver more effective treatment. The duration of action of the drug in the patient's body is usually limited, mainly due to the incompatibility of the drug with the human body. To resolve this issue, it is necessary to establish a connection between the patient and the therapeutic drug, which will allow to set up a compatible system before starting the therapeutic treatment. A compatible system could be created by implementing the concept of personalised therapy, where the drug is adapted to and linked with the patient's lifestyle preferences. For example, some patients cannot tolerate meat or meat-derived ingredients. Therefore, it is necessary to ensure that the drug is free of animal ingredients, and to establish the link between the drug and the patient, the drug is labelled as vegan. Similarly, drugs can be personalised and labelled as kosher for Jews and halal for Muslims [32]. Some patients are sensitive to certain substances such as sugar, lactose or gluten. It is therefore important to ensure that these sensitive ingredients are not included in the formulation, thus it is necessary to verify that the manufacturing process and ingredients of the drug are compatible with the patient's lifestyle [33]. Favourable circumstances for recovery are created by integrating more than one effect into the treatment process (Figure 2). It is obvious that a drug alone will not lead to a complete recovery, but a multiple effects have the potential to activate the synergistic effect and could lead to a more effective treatment. Thus, a combination of effects can be achieved by providing a drug that is compatible with the patient's lifestyle. A compatible system enhances the trust in the drug, which in turn triggers the placebo effect. Despite the fact that a qualified and rationally designed drug is administered, which potentially has a therapeutic effect, the third effect emerges from the homogeneous effect resulting from the implementation of the harmonized system, which in turn maximizes the cause-effect information and finally activates the potential energy. Eventually, the total effect in the body resulting from the drug effect, the placebo effect and the harmonized effect are usually higher than the sum of all effects. The coordination between all the effects activates the synergistic effect, which acts as an outsourcing effect, thus it is likely that the total effect, including the synergistic effect, can act as a source of a "miraculous" cure that promotes the healing process towards a complete recovery.

The tolerance system created by the compatibility between therapy and mind can often lead to viewing the therapeutic drug as a compatible entity; thus, reducing rejection mechanisms and prolonging the duration of action, which contributes to inducing more effective treatments. Further research and studies are needed to understand the role of entropy in disease development [34]. Additional research is needed to explore the role of the compatible system in improving body-mind communication, which in turn could improve signalling between cancer cells and the brain and possibly reactivate the immune system to find and destroy tumor cells. A new therapeutic program needs to be initiated that implements the proposed favourable circumstances for healing and targets entropy as a source of energy for disease development and prevention.

Citation: Jawad Alzeer. "Halalopathy: Revival of Miraculous Cure and Creation of Favourable Circumstances for Cancer Therapy". Medicon Medical Sciences 2.3 (2022): 21-28. 


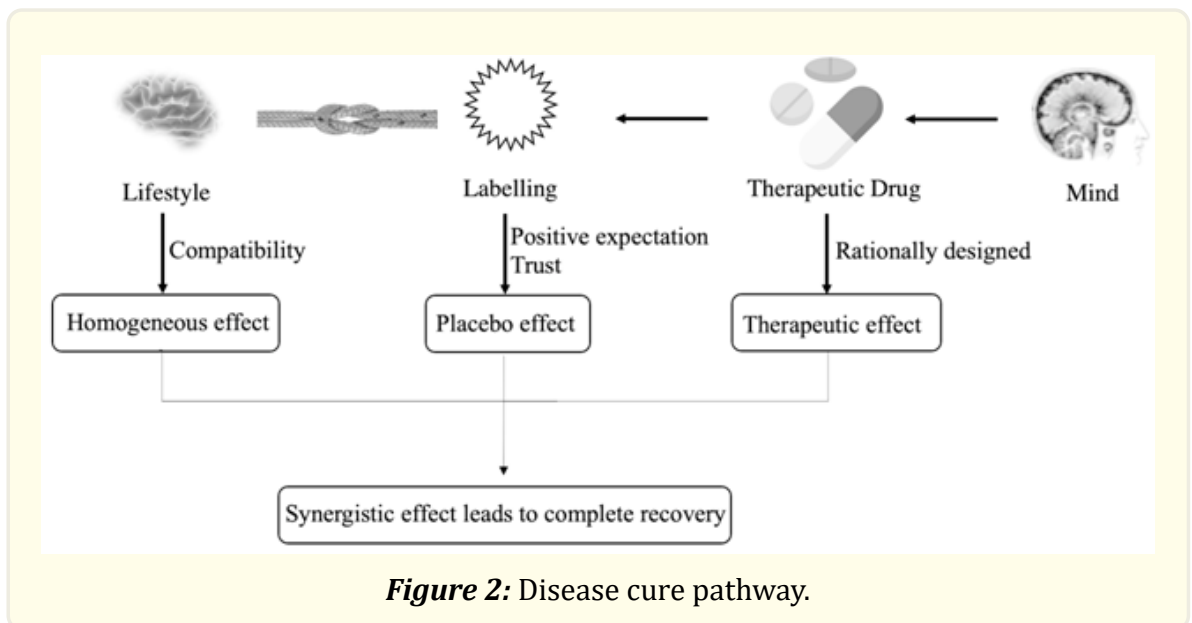

\section{Conclusions}

Seeking a complete recovery in cancer therapy is demanding and challenging, however understanding the favourable circumstances for a cure could drive the therapeutic process towards a complete recovery. The evaluation of reports on "miraculous" cures has shown that cancer patients who achieved a complete recovery were usually not dependent solely on the therapeutic effect of chemotherapy, but indeed they explored more than one effect to speed-up the healing process; building trust in the process and enhanced confidence in the patient's personality can potentially lead to a complete recovery. Halalopathy has already concluded that favourable circumstances are crucial for healing, yet this can be achieved by building a compatible system between the patient and the therapeutic process, thereby conceivably triggering a coordination effect. The placebo effect, the therapeutic effect, and the enhanced cause-effect information could activate the synergistic the effect, which could act as an additional source of effect, driving the "miraculous" cure towards complete recovery.

\section{Acknowledgments}

We would like to thank Prof. Khaled Abou Hadeed and Khaled Al-Bakri for useful discussion and support.

\section{Footnote}

\section{Conflicts of interest}

The authors declare that they have no conflicts of interest.

\section{Funding}

None.

\section{Ethical Statement}

The authors are accountable for all aspects of the work in ensuring that questions related to the accuracy or integrity of any part of the work are appropriately investigated and resolved.

\section{References}

1. Hoda M. "Potential Alternatives to Conventional Cancer Therapeutic Approaches: The Way Forward". Curr Pharm Biotechnol 22.9 (2021): 1141-1148. 
2. Shinall MC Jr, Stahl D and Bibler TM. “Addressing a Patient's Hope for a Miracle”. J Pain Symptom Manage 55.2 (2018): 535-539.

3. Rediger J. Cure: The remarkable science and stories of spontaneous healing and recovery, Penguin Books Ltd. London, United Kingdom (2020).

4. Vitry AI, Shin NH and Vitre P. "Assessment of the therapeutic value of new medicines marketed in Australia”. J Pharm Policy Pract 6 (2013): 2.

5. Tewabe A., et al. "Targeted Drug Delivery - From Magic Bullet to Nanomedicine: Principles, Challenges, and Future Perspectives". J MultidiscipHealthc 14 (2021): 1711-1724.

6. Schcolnik-Cabrera A., et al. "Understanding tumor anabolism and patient catabolism in cancer-associated cachexia”. Am J Cancer Res 7.5 (2017): 1107-1135.

7. Alzeer J. "Entropy and potential energy as a key role of halalopathy in disease prevention and cure". Longhua Chin Med 3 (2020): 20.

8. Kotas ME and Medzhitov R. "Homeostasis, inflammation, and disease susceptibility". Cell 160.5 (2015): 816-827.

9. Salleh MR. "Life event, stress and illness". Malays J Med Sci 15.4 (2008): 9-18.

10. Mehlisch DR. "Narcotic agonist-antagonist: a brief historical review with clinical application”. Clin Res Pract Drug Regulatory Aff 4 (1986): 367-379.

11. Lajtha A and Vizi ES. Handbook of neurochemistry and molecular neurobiology neurotransmitter systems. Springer, New York (2008).

12. Filatova EV, Shadrina MI and Slominsky PA. "Major Depression: One Brain, One Disease, One Set of Intertwined Processes”. Cells 10.6 (2021): 1283.

13. Niedzwiedz CL., et al. "Depression and anxiety among people living with and beyond cancer: a growing clinical and research priority". BMC Cancer 19 (2019): 943.

14. Reiche EM, Nunes SO and Morimoto HK. "Stress, depression, the immune system, and cancer". Lancet Oncol 5.10 (2004): 617-25.

15. Segerstrom SC and Miller GE. "Psychological stress and the human immune system: a meta-analytic study of 30 years of inquiry". Psychol Bull 130.4 (2004): 601-630.

16. Correia AS, Cardoso A and Vale N. “Highlighting Immune System and Stress in Major Depressive Disorder, Parkinson's, and Alzheimer's Diseases, with a Connection with Serotonin". International Journal of Molecular Sciences 22.16 (2021): 8525.

17. Masih J, Belschak F and Verbeke JMIW. "Mood configurations and their relationship to immune system responses: Exploring the relationship between moods, immune system responses, thyroid hormones, and social support". PLoS One 14.5 (2019): e0216232.

18. Wang Z, Wu Z, Liu Y and Han W. "New development in CAR-T cell therapy”. J Hematol Oncol 10.1 (2017): 53.

19. Evers R., et al. "Disease-Associated Changes in Drug Transporters May Impact the Pharmacokinetics and/or Toxicity of Drugs: A White Paper from the International Transporter Consortium”. Clin PharmacolTher 104.5 (2018): 900-915.

20. Kunnumakkara AB., et al. “Cancer drug development: The missing links”. Exp Biol Med 244.8 (2019): 663-689.

21. Lagman RA, Yoo GJ, Levine EG, Donnell KA, Lim HR. "Leaving it to God” religion and spirituality among Filipina immigrant breast cancer survivors. J Relig Health 53.2 (2014): 449-460.

22. Boehmer T. "Miracle survivors: beating the odds of incurable cancer". Skyhorse publishing (2014).

23. Alzeer J. "Halalopathy: A science of trust in medicine". J Integr Med 17.3 (2019): 150-154.

24. Alzeer J. "Halalopathic: a new concept in medicine". J Mol Genet Med 12 (2018): 353.

25. Meier RPH., et al. "Immunologic Clearance of a BK Virus-associated Metastatic Renal Allograft Carcinoma". Transplantation 105.2 (2021): 423-429.

26. Naser AY., et al. "Depression and anxiety in patients with cancer: a cross-sectional study". Front. Psychol 12 (2021): 585534.

27. Stark DP and House A. “Anxiety in cancer patients”. Br J Cancer 83.10 (2000): 1261-1267.

28. Pitman A, Suleman S, Hyde N and Hodgkiss A. "Depression and anxiety in patients with cancer”. BMJ 361 (2018): k1415.

29. Tarabichi M., et al. Systems biology of cancer: entropy, disorder, and selection-driven evolution to independence, invasion and 
"swarm intelligence". Cancer Metastasis Rev 32 (2013): 403-421.

30. Abbott M and Ustoyev Y. "Cancer and the Immune System: The History and Background of Immunotherapy". Semin Oncol Nurs 35.5 (2019): 150923.

31. Garg AD and Agostinis P. "Cell death and immunity in cancer: From danger signals to mimicry of pathogen defense responses". Immunol Rev 280.1 (2017): 126-148.

32. Alzeer J. "Permissible Medicine and Rationalization of Halal Pharma”. Halalpshere 1.1 (2021): 43-52.

33. Alzeer J and Hadeed K.A. "Halal Certification of Food, Nutraceuticals, and Pharmaceuticals in the Arab World". Laher I. (eds) Handbook of Healthcare in the Arab World. Springer, Cham (2020).

34. Hanselmann RG and Welter C. “Origin of Cancer: An Information, Energy, and Matter Disease”. Front. Cell Dev. Biol 17 (2016): $1-12$.

Volume 2 Issue 3 March 2022

(C) All rights are reserved by Jawad Alzeer. 